\title{
Surface treatment-controlled solvothermal synthesis of highly active reduced 1D titania with heterojunctioned carbon allotrope
}

\author{
Ahmed Badreldin $^{1} \cdot$ Yahya Zakaria ${ }^{2} \cdot$ Said Mansour ${ }^{2} \cdot$ Ahmed Abdel-Wahab $^{1}$ (it)
}

Received: 10 November 2020 / Accepted: 11 January 2021 / Published online: 20 January 2021

(C) The Author(s) 2021

\begin{abstract}
One-dimensional (1D) nanowire black titania heterojunctioned with multi-wall carbon nanotube $\left(\mathrm{bTiO}_{2} \mathrm{NW} / \mathrm{MWCNT}\right)$ structures were successfully synthesized via a facile single-step hydrothermal procedure, coupled with succeeding surface treatments and a solid-state physiochemical mode of reduction. Paramagnetic $\mathrm{SiO}_{2}$-coated $\mathrm{Fe}_{3} \mathrm{O}_{4}$ microspheres were fabricated and used as cores for the seeding and growth of the $\mathrm{bTiO}_{2} \mathrm{NW} / \mathrm{MWCNT}$ photocatalyst. The as-prepared photocatalysts were characterized via X-ray diffraction, scanning electron microscopy, transmission electron microscopy, X-ray photoemission spectroscopy, Fourier transform infrared spectroscopy, and UV-vis diffuse reflectance spectroscopy. The results of materials characterization confirmed formation of $1 \mathrm{D} \mathrm{bTiO}_{2} \mathrm{NW}$ structure with chemically bound MWCNT atop the reduced $\mathrm{Ti}^{3+}$ propagated lattice of the predominantly (101) exposed facets of anatase $\mathrm{TiO}_{2}$. Controlling the surface treatment process and NW growth time to maintain the anatase phase and stability of surface morphology upon reduction allowed for superior visible light-driven photoactivity. The visible light-driven photocatalytic degradation of $10 \mathrm{mg} / \mathrm{L}$ methylene orange was recorded at $97.4 \%$ in 20 min of 0.7 Sun intensity. The apparent reaction rate constant $(\mathrm{k})$ of the as-prepared photocatalyst $\left(0.1439 \mathrm{~min}^{-1}\right)$ is $\sim 18$ times higher than that of pristine $\mathrm{TiO}_{2}$. Utilization of paramagnetic cores for in situ photocatalyst collection upon water treatment is highly recommended for newly developed materials. Further, performing surface treatment procedures of prepared titania-based photocatalysts has been proven to have a notable advantageous effect on photoactivity and is thus suggested for similar materials.
\end{abstract}

Keywords Photocatalysis $\cdot$ Black titania $\cdot \mathrm{NaBH}_{4}$ reduction $\cdot$ Surface treatment $\cdot \mathrm{Ti}^{3+}$

\section{Introduction}

One of the recent emerging areas of contaminant degradation is heterogeneous photocatalysis, which has seen a markedly swell of interest due to abundance and relative cost-effectiveness of semi-conductor materials employed and ability to degrade contaminants in situ. Briefly, a photocatalytic process involves a reaction that is preliminarily activated by the absorption of a photon with ample energy to overcome the bandgap energy of the photoactive material used. The bandgap energy is in essence the difference in energy between the valence band (VB) - where the semi-conductor's valence electron is in its ground state and

Ahmed Abdel-Wahab

ahmed.wahab@qatar.tamu.edu

1 Chemical Engineering Program, Texas A\&M University at Qatar, P.O. Box 23874, Doha, Qatar

2 Qatar Environment and Energy Research Institute, P. O. Box 34110, Doha, Qatar the conduction band (CB), which is the higher energy level the electron, temporarily resides about during photoexcitation. Upon photoexcitation, the electron leaves a "hole" at the VB whereby allowing the generation of oxidizing radicals. Generally, in water systems, the photoexcited electron reacts with diatomic oxygen - either as part of the dissolved oxygen in water or from surface oxygen - to produce superoxide radical species $\left(\mathrm{O}_{2}^{-}{ }^{-}\right)$. At the same time, the generated hole either oxidizes an organic contaminant or reacts with available water molecules resulting in hydroxide radical $(\cdot \mathrm{OH})$ which can easily oxidize organic contaminants. Thus, it is during the intermittent time that the electron is at the CB that photodegradation of a contaminant is feasible due to the near-simultaneous generation of oxidizing radicals. However, depending on the photocatalytic material employed, the electron/hole recombination rate varies - presenting a prominent challenge in the case where the recombination rate is high. Simply, in the scenario that the recombination rate exceeds the time it takes to generate an oxidizing radical, the photoexcitation process is essentially wasted, subsequently decreasing the apparent photoactivity and quantum efficiency of the photocatalyst. 
Titanium dioxide has perhaps been the single-most investigated and developed photocatalyst owing to several unique characteristics. Its exceptional photo and chemical stability, relative abundance, and nontoxicity have promoted its use in a myriad of different applications over the years $[1,2]$. However, certain intrinsic properties of $\mathrm{TiO}_{2}$ render a limit to its photocatalytic efficiency (PCE) with organic contaminant degradation. Specifically, the relatively low adsorptive capacity and surface area limit the amount of active adsorption sites available for a photodegradation phenomenon. Moreover, $\mathrm{TiO}_{2}$ has a bandgap energy of approximately $3.2 \mathrm{eV}$ for anatase and $3.0 \mathrm{eV}$ for rutile [3]. This is a fairly high bandgap energy which amounts to only $3-5 \%$ of solar photons having enough energy to initiate a photoexcitation event. The high degrees of essentially redundant photons that continue to bombard the semi-conductor during solar irradiation are simply wasted. Innately, $\mathrm{TiO}_{2}$ has a disadvantageously high electron-hole recombination rate which, as previously mentioned, significantly reduces its PCE and quantum efficiency. Although the aforementioned drawbacks are significant, researchers have explored modification techniques and synthesis pathways of different materials to tackle them. Unfortunately, due to the complex effects that may be introduced when a material is modified, it is not uncommon for an advantageous change in one of those drawbacks to negatively affect another property detrimental to the photocatalytic process. A quite significant factor that is detrimental in the practical utilization of nanocomposite in water treatment systems is the prerequisite of allowing for a retainment mechanism of the NP composite. Therefore, separating the active photocatalyst composite from the treated water and then retaining them in the photoreactor for further use is of paramount importance to the feasibility of the process, and to ensure the cyclability of the $\mathrm{TiO}_{2}$-based photocatalyst is being utilized appropriately and sustainably. Some work has been done with photocatalysts being coated upon a paramagnetic iron core which can be collected when passed through a magnetic field [4-6].

In order to fabricate a highly photoactive material, the brevity and complexity of a multitude of parameters need to be fine-tuned simultaneously and effectively in order to achieve expedient results. Over the years, several techniques have been developed to undertake these impediments such as coupling with other semi-conductors, combining with carbonbased materials, modification with metals and/or metal oxides, and cation or anion doping [7-10]. However, these modifications were found to have slight effects in extending the spectral response of $\mathrm{TiO}_{2}$ materials to visible light; therefore, most visible light and infrared (IR) energy is still wasted as previously noted. A more substantial utilization of the more available visible light segment of the solar spectrum was achieved via the serendipitous discovery of black $\mathrm{TiO}_{2}$ in 2011 via high pressure hydrogenation synthesis [11]. Since its initial discovery, several more environmentally benign techniques were employed to reduce the ground state $\mathrm{Ti}^{4+}$ to its $\mathrm{Ti}^{3+}$ reduced state [12]. Other chemical oxidation [13] and electrochemical reduction [14] methods have also been investigated and black $\mathrm{TiO}_{2}$ nanomaterials have successfully been fabricated from both. Significant work has been undertaken on oxygen vacancy engineering towards an array of photo and electrocatalytic materials in recent years $[15,16]$.

In tackling the challenge of a high electron-hole recombination rate of $\mathrm{TiO}_{2}$, it has been postulated that the addition of a high electron acceptor, such as carbon nanotubes (CNTs), should promote electron-hole separation, effectively decreasing the electron-hole recombination rate. This in turn will allow for a more relaxed time for the photoexcited electron to be accepted by the skeletal CNTs and travel to the surface, thereby allowing for a higher probability of photocatalytic degradation of an organic contaminant. This in turn leads to an effective higher photocatalytic efficiency $[11,17]$. Due to the advantageous properties of CNTs, several reported works have shown its incorporation with different morphological $\mathrm{TiO}_{2}$ structures and under different procedures such as mechanical mixing of $\mathrm{TiO}_{2}$ and CNTs [18], sol-gel synthesis of $\mathrm{TiO}_{2}$ in the presence of CNTs [19], electrospinning methods [20], and hydrothermal [21] and chemical vapor deposition [22].

Among the most prominent design challenges of $\mathrm{TiO}_{2}$ nanoparticle or 1-dimensional fibrous morphologies, achieving and maintaining a predominant anatase crystalline phase with (101) exposed facets while comfortably manipulating the morphology, heterojunctioning with electron acceptors, and controlling surface defects and oxygen vacancies in black titania has been a challenge. The (101) exposed anatase facet has been deemed most preferable for a high photoactive sample due to the high concentration of surface defects, compared with other $\mathrm{TiO}_{2}$ crystalline phases. This can be rather advantageous in the generation of more oxygen vacancies (i.e., during reduction schemes of pristine to black titania) which can act as electron sinks in effect further reducing the electronhole recombination rate. Moreover, owing to the (101) anatase facet yielding the lowest surface energy $\left(0.44 \mathrm{~J} / \mathrm{m}^{2}\right)$, it further substantiates its strong reduction capabilities.

In the present study, the aim is to effectively design a tailored black titania-based photocatalyst with a 1-dimensional nanowire morphology that is effectively heterojunctioned to multiwalled carbon nanotubes (MWCNTs). Photodegradation performance experiments will be conducted on the photoactive products of incremental synthesis steps to probe a clearer understanding on the systematic enhancement of photoactivity. The effect of synthesis variations including, but not limited to, use of templating agents in nanowire growth, surface treatment procedures, reduction schemes, and titania precursor for MWCNT incorporation to the photocatalyst mesh will all be investigated to determine their effect on the apparent 
photodegradation of methylene orange (MO) in aqueous solution as an organic contaminant mimicking agent in water systems. The optimized photocatalyst design will also be synthesized, and its photodegradation performance examined, atop a paramagnetic iron oxide core for ease of retainment upon complete photodegradation of the contaminants.

\section{Materials and method}

\subsection{Materials}

Titanium isopropoxide (TIP, >98\%), isopropanol, P25 (21$\mathrm{nm}$ primary particle size (TEM), $>99.5 \%$ trace metal basis), tetraethyl orthosilicate (TEOS, reagent grade, 98\%), multiwalled carbon nanotubes (MWCNT, >98\% carbon basis), methyl orange ( $\mathrm{MO}, \mathrm{C}_{14} \mathrm{H}_{14} \mathrm{~N}_{3} \mathrm{NaO}_{3} \mathrm{~S}$, ACS reagent, 85\%), humic acid sodium salt (HA, technical grade), sodium hydroxide $(\mathrm{NaOH}, \mathrm{ACS}$ reagent, $>97 \%$ pellets), and methylene blue (MB, $\mathrm{C}_{16} \mathrm{H}_{18} \mathrm{ClN}_{3} \mathrm{~S}$ ) were purchased from Sigma-Aldrich Co. (St. Louis, MI, USA). Sodium borohydride $\left(\mathrm{NaBH}_{4}\right)$ was purchased from Alfa Aesar. Throughout all experiments, deionized water was attained through the Millipore $(18.2 \mathrm{M} \Omega \mathrm{cm})$ purified water system.

\subsection{Catalyst preparation}

\subsubsection{Synthesis of pristine titania nanowires with MWCNT (pTNW@MWCNT)}

Commercial Aeroxide® P25 with 21-nm primary particle size was purchased from Sigma-Aldrich. Pristine TiO2 nanowires with incorporated MWCNTs (pTNW/MWCNT) were synthesized using a modified procedure adopted from Rao et al. [23]. Briefly, $1.0 \mathrm{~g}$ of $\mathrm{P} 25$ powder and $5 \mathrm{w} \%$ MWCNT were added to aqueous $\mathrm{NaOH}(70 \mathrm{~mL}, 10 \mathrm{M})$ solution under vigorous stirring in a $100-\mathrm{mL}$ Pyrex beaker for $15 \mathrm{~min}$ to obtain a homogenous suspension of $\mathrm{TiO} 2$ nanoparticles (NPs) with the MWCNTs. The mixture was quickly added to a Teflonlined container ( $30 \%$ headspace by volume) and enclosed in a stainless-steel autoclave. Titania nanowires synthesized with ethanol as a templating agent were fabricated using an equivolume mixture of ethanol and $\mathrm{NaOH}(10 \mathrm{M})$. A solvothermal reaction was then facilitated by keeping the autoclave in a thermostatic oven at $180^{\circ} \mathrm{C}$ for $20 \mathrm{~h}$. The resultant grayish pulp was washed and centrifuged at 6000 RMP with $0.1 \mathrm{M} \mathrm{HCl}$ and DI water several times. The pulp was then vacuum dried prior to surface treatment (discussed below), or directly calcinated at $450{ }^{\circ} \mathrm{C}$ for $4 \mathrm{~h}$. Using a higher calcination temperature can result in an unfavorable phase shift and more importantly can cause combustion of MWCNTs.
Surface treatment of selected samples was performed after the final morphological modification and prior to the calcination step that was adapted from Tian et al. with some modification [24]. After complete washing of sample, they were stirred in $0.5 \mathrm{M} \mathrm{HCl}$ for $24 \mathrm{~h}$. This was followed by a thorough washing with DI water. The samples were then isolated by centrifugation at 6000 RPM and vigorously stirred in $70 \mathrm{~mL}$ of diluted sulfuric acid $(0.05 \mathrm{M})$ for $30 \mathrm{~min}$. Upon reaching a homogenous mixture, the solution was transferred to a Teflonlined stainless-steel autoclave and heated to $120{ }^{\circ} \mathrm{C}$ for $4 \mathrm{~h}$. Samples that underwent this surface treatment step were then calcinated at $450{ }^{\circ} \mathrm{C}$ for $4 \mathrm{~h}$.

\subsubsection{Reduction to "black" titania nanowires with MWCNT (bTNW@MWCNT)}

All titania-based nanocomposites were effectively reduced (to black titania-based) through a simple mechanochemical reduction step using sodium borohydride $\left(\mathrm{NaBH}_{4}\right)$ [25]. An optimized molar amount of $\mathrm{NaBH}_{4}$ :titania of $1.25: 1$ was collected and thoroughly mixed for 10 min using an Agate pestle and mortar. The powder mixture was transferred to a ceramic crucible and placed in a tubular furnace. Prior to heating, the system was vacuumed to remove any oxygen in the reactor's volume and then purged with Argon until atmospheric pressure was restored. Reduction was carried out at $380^{\circ} \mathrm{C}$ for $2 \mathrm{~h}$ following 1-h heating time. The reaction was facilitated under $150 \mathrm{sccm}$ of Argon flow. The sample was cooled to room temperature prior to opening the reactor and extracting the crucible. This procedure allowed all unreacted $\mathrm{NaBH}_{4}$ to cool to room temperature in order to allow safe handling during the following washing step [26]. The as-prepared samples were then washed and centrifuged several times using DI water and ethanol. Finally, the collected samples were vacuum dried overnight at $80^{\circ} \mathrm{C}$.

\subsubsection{Incorporation of silica-coated paramagnetic iron core for shell bTNW@MWCNT}

With regard to fabricating the paramagnetic silica-coated iron oxide core, a refined synthesis scheme to that of $\mathrm{Yu}$ et al. was employed [27]. First, fabricating paramagnetic $\mathrm{Fe}_{3} \mathrm{O}_{4}$ microspheres was performed using a hydrothermal procedure. Then, using a modified Stöber methodology, an amorphous, inert, and thermally stable silica coating was introduced atop the iron core at ambient conditions [28]. Growing pTNW atop the $\mathrm{Fe}_{3} \mathrm{O}_{4} @ \mathrm{SiO}_{2}$ paramagnetic was achieved through a twostep process. First, depositing pTNP with high surface coverage was achieved by dispersing $\mathrm{Fe}_{3} \mathrm{O}_{4} @ \mathrm{SiO}_{2}(1 \mathrm{~g})$ in wellstirred solution of IPA (70 mL), dissolved EDTA (0.25 g), and $250 \mu \mathrm{L}$ of TIIP. Stirring was continued for $30 \mathrm{~min}$ at room temperature; then the solution was transferred to a $100-\mathrm{mL}$ Teflon-lined stainless-steel autoclave and maintained at 
$150{ }^{\circ} \mathrm{C}$ for $24 \mathrm{~h}$. The autoclave was then cooled to room temperature. The brown-black pulp was collected via a small magnet, washed and centrifuged several times with DI water and ethanol, and then air dried overnight yielding pTNP@ $\mathrm{Fe}_{3} \mathrm{O}_{4} @ \mathrm{SiO}_{2}$. In order to facilitate titania nanowire growth, $2 \mathrm{~g}$ of the as-prepared sample was mixed in $\mathrm{NaOH}$ $(70 \mathrm{~mL}, 10 \mathrm{M})$ solution, and $5 \mathrm{w} \%$ MWCNT were added. The nanowire growth procedure previously discussed was undertaken. In order to maintain a high surface coverage in the final TNW growth on the silica surface, $1.5 \mathrm{~g}$ of commercial grade P25 was added to the $\mathrm{NaOH}$ solution prior to transferring it to the autoclave. Obtaining the reduced black TNW/ MWCNT@ $\mathrm{Fe}_{3} \mathrm{O}_{4} @ \mathrm{SiO}_{2}$ followed the same mechanochemical process discussed previously.

\subsection{Catalyst characterization}

In order to effectively characterize the morphology and structure of the different samples prepared through the different synthesis methods, both a scanning electron microscope (SEM, FEI Quanta 400 FEG ESEM) and transmission electron microscopy (TEM, JEOL JEM-2100 FEG TEM) were employed. In order to enhance sample stability during high intensity electron beam exposures of SEM/TEM, the asprepared samples were gold-coated under Argon, as the process gas, in order to enable higher resolution imaging. This was performed using a sputtering device (LEICA, EM SCD050). EDX and elemental mapping were performed via both integrated SEM and TEM EDX devices. Crystallinity was measured via XRD analysis (Rigaku, Ultima IV) at a $2 \theta$ angle scanning rate of $1 \% / \mathrm{min}$. Surface analysis has been conducted using X-ray photoelectron spectroscopy (XPS) (ESCALAB 250Xi, Thermo Fisher Scientific) equipped with monochromate Al K-alpha X-ray source at $1486.6 \mathrm{eV}$. The pass energy for high-resolution scans was $20 \mathrm{eV}$ and for survey scan was $100 \mathrm{eV}$. UV-Vis-NIR diffusion reflectance spectra were realized using a solid-sample unit on a PerkinElmer Lambda 950 Spectrophotometer, which allowed for bandgap energy estimation of the photocatalysts prepared. FTIR spectrophotometry analysis was used for functional group identification (Varian 640-IR). The spectral range of the obtained spectra was between 4000 and $450 \mathrm{~cm}^{-1}$ with a resolution of $4 \mathrm{~cm}^{-1}$.

\subsection{Evaluation of photocatalytic degradation efficiency}

The photocatalytic performance of the as-prepared materials was characterized by evaluating material's performance for methylene orange $(\mathrm{MO})$ degradation in a batch reactor system. A $50 \mathrm{~mL}$ of $10 \mathrm{mg} / \mathrm{L} \mathrm{MO}$ aqueous solution was used for each experimental run, with a photocatalyst dose of $1 \mathrm{~g} / \mathrm{L}$. The mixture was stirred for $30 \mathrm{~min}$ in the dark to ensure an adsorption/desorption equilibrium. Upon artificial solar irradiation $\left(700 \mathrm{~W} / \mathrm{m}^{2}\right)$ with a solar simulator calibrated using a standard pyranometer (CMP6 Kipp \& Zonen with a spectral range of 285-2800 nm), samples were taken at the desired reaction times using a $10-\mathrm{mL}$ disposable syringe and immediately filtered using a $0.45 \mu \mathrm{m}$ mixed cellulose ester filter paper obtained from Advantec. The filtered aliquots were stored in 10 -mL scintillated glass vials until analysis for MO using UVVis-NIR spectrometer (PerkinElmer 950 Spectrophotometer).

Briefly, the absorption spectra for each liquid sample were obtained between 400 and $550 \mathrm{~nm}$, and the maximum absorption of MO was recorded at $464 \mathrm{~nm}$. Effectively, this allows quantification of the remaining MO residue present at different sampling times, in turn allowing to calculate the percentage MO degraded at different irradiation times, with different photocatalysts. The percentage degradation can thus simply be calculated using Eq. (1) below:

Degradation $(\%)=\frac{100 *\left(\mathrm{C}_{\mathrm{o}}-\mathrm{C}_{\mathrm{t}}\right)}{C_{0}}$

where $\mathrm{C}_{0}(\mathrm{mg} / \mathrm{L})$ is the initial MO concentration and $\mathrm{C}_{\mathrm{t}}(\mathrm{mg} /$ $\mathrm{L})$ is the concentration of $\mathrm{MO}$ after time ( $\mathrm{t}$ ( $\mathrm{min}$ ).

\section{Results and discussion}

\subsection{Effects of synthesis procedure and doping on catalyst characteristics}

\subsection{1 $\mathrm{TiO}_{2}$ Crystallinity, average crystallite size, and morphology}

X-ray diffraction (XRD) patterns of the samples are shown in Fig. 1. Figure 1a shows a distinct difference in crystallinity distribution between the anatase and rutile phases of the pristine titania nanowires (pTNW), whereby the use of ethanol as a templating agent in the hydrothermal NW growth route shows a complete phase transformation from a predominant anatase-present in commercial P25 used as the pTNW precursor - to a complete rutile phase shift. Performing the hydrothermal growth without templating ethanol allows for an approximate 50\% maintenance of the original photoactive anatase phase. Recorded peaks corresponding to the (101), (004), (200), (105), (211), (204), and (215) reflections are indexed to anatase crystallites in titania (PDF no. 21-1272) [29]. In order to discern the contributing phase effects of the hydrothermal process and the calcination step, respectively, we characterized the phases of pTNW fabricated hydrothermally for $20 \mathrm{~h}$ without the templating ethanol, surface treatment, or calcination (pTNW (20 h, no EtOH, no st, no calcination)) and its counterpart (pTNW (20 h, no EtOH, no st, calcination)) which has been calcinated. We identified the 

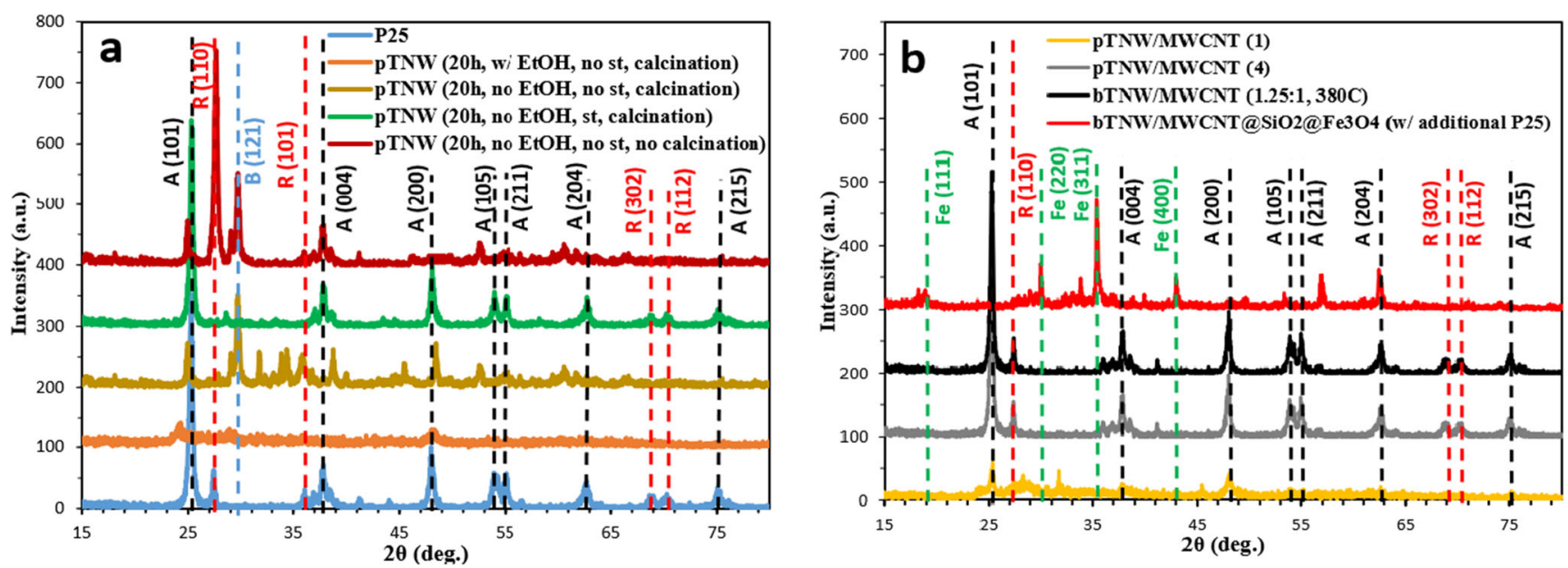

Fig. 1 X-ray diffraction (XRD) patterns of all as-prepared photocatalytic samples. Peaks are labeled with "A" for anatase, "R" for rutile, "B" for brookite, and " $\mathrm{Fe}$ " for $\mathrm{Fe}_{3} \mathrm{O}_{4}$ phases

(121) phase of brookite $\mathrm{TiO}_{2}$ at $29.8^{\circ}$ in both samples, indicating that the surface treatment eliminates this phase. Nonetheless, a notable reduction in peak intensities for the anatase phase is observed for the un-calcinated sample. This confirms the effect of calcination on the anatase phase transformation during synthesis of hydrothermally fabricated catalysts. The mildly acidic surface treatment procedure undertaken on the as-synthesized pTNW, fabricated without ethanol assisted growth, prior to calcination, actually enhances the anatase phase composition to yield a full anatase phase titania sample. The same surface treatment procedure was tested when facilitating pTNW growth with the addition of MWCNT. To effectively investigate heterojunctioning between the pTNW and MWCNT, two variations in synthesis were performed: (1) using P25 and pTNW as titania precursors prior to hydrothermal procedure of MWCNT incorporation and (2) surface treatment prior to calcination of nanocomposite. Figure $1 \mathrm{~b}$ shows that an enhanced anatase phase is achieved for pTNW/MWCNT (4) which underwent surface treatment and was fabricated with pTNW as the titania precursor. The reduction procedure optimized to yield the most stable and photoactive black titania-based sample showed that a significant phase transformation is not to be expected during reduction, thus maintaining a $90 \%$ anatase phase for the bTNW/MWCNT sample. XRD peaks of the final reduced paramagnetic nanocomposite-bTNW/ MWCNT@SiO $@ \mathrm{Fe}_{3} \mathrm{O}_{4}$ — show a slight absence or reduction in characteristic anatase titania peaks, indicating that the nanocrystalline titania structures are well dispersed in the overall composite mesh. Therefore, the absence of ethanol as a templating agent, presence of calcination, and surface treatment allowed for a thorough anatase phase formation. Phase transition was found to be largely dependent on the calcination step which allows for favorable phase transformation to the more photoactive anatase. The Scherrer equation was employed to approximate the average crystallite size of samples [30]. Scherrer approximations show about $50 \%$ reduction in average crystallite size of the former as indicated in Table 1. A larger crystallite size is innately expected to increase the migration distance of a photoexcited electron prior to reach
Table 1 Summary of percentage phase composition and average crystallite size using Scherrer approximation

\begin{tabular}{llll}
\hline Sample & \multicolumn{2}{l}{ Phase (\%) } & \multirow{2}{*}{ Avg. crystallite size (nm) } \\
\cline { 2 - 3 } & Anatase & Rutile & \\
\hline P25 & 90.8 & 9.2 & 17.19 \\
pTNW (20 h, w/ EtOH, no st, calcination) & - & 100 & 9.34 \\
pTNW (20 h, no EtOH, no st, calcination) & 49.1 & 50.9 & 18.32 \\
pTNW (20 h, no EtOH, st, calcination) & 100 & - & 19.68 \\
pTNW (20 h, no EtOH, no st, no calcination) & 18.4 & 72.7 & 11.43 \\
pTNW/MWCNT (1) & 81.7 & 18.3 & 3.49 \\
pTNW/MWCNT (4) & 91.3 & 8.7 & 19.78 \\
bTNW/MWCNT & 90.4 & 9.6 & 19.42 \\
bTNW/MWCNT@SiO2@Fe3O4 (w/ P25 addition) & - & - & - \\
\hline
\end{tabular}


the surface of its parent titania crystal. This in turn increases the probability of a successful photochemical reaction induced by the electron-hole redox properties and favorably decreases the electron-hole recombination rate.

SEM images in Fig. 2a and b depict the surface effects that profoundly alter the pTNW morphology. The use of ethanol as a templating agent is seen to produce lengthier strands of intertwined nanowires, composed of stacked nanorods with approximately $70 \mathrm{~nm}$ axial lengths. Characteristically crystalline nanowire shards with varying axial lengths are generated when a purely alkaline hydrothermal medium is utilized for nanowire growth, coupled with the surface roughening mildly acidic surface treatment procedure prior to calcination. Figure $2 \mathrm{c}$ and $\mathrm{d}$ show the nano-spherical composite of pristine titania nanoparticles (pTNP) hydrolyzed atop the silica-coated
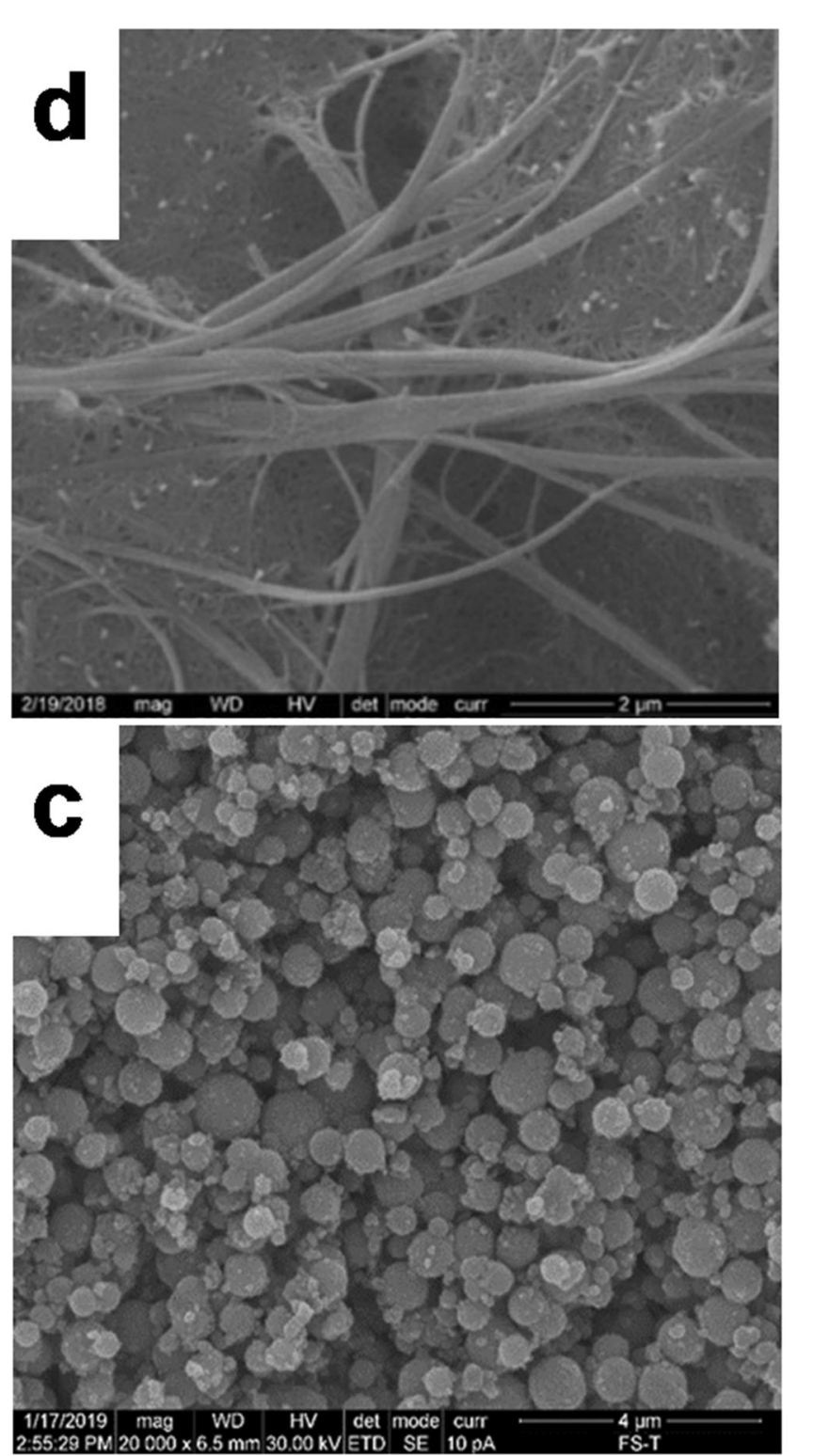

Fig. 2 Scanning electron microscopy (SEM) images: a pTNW (20 h, w/ EtOH, no st, calcination); b pTNW (20 h, no EtOH, st, calcination); c pTNP@SiO $\mathrm{Si}_{2} @ \mathrm{Fe}_{3} \mathrm{O}_{4} ; \mathbf{d}$ pTNW@ $\mathrm{SiO}_{2} @ \mathrm{Fe}_{3} \mathrm{O}_{4}$ (w/ additional P25)

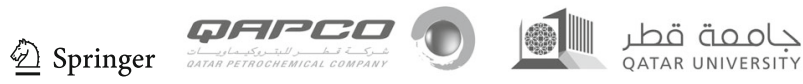

paramagnetic core, and the maintained morphological features of spherical paramagnetic cores intertwined with lengthy crystalline pTNW. SEM image in Fig. $2 d$ is for the sample that included the addition of extra P25 prior to NW hydrothermal growth scheme in order to ensure better surface coverage of the titania nanowires atop the surface of the paramagnetic core.

TEM images in Fig. 3a and $b$ show a clear surface distinction atop the titania nanowires, whereby the treated nanowires exhibit roughened surface edges with fewer titania nanoshreds populating the surface. The untreated sample shows a clear stacking of titania nanowires; thus, the roughened edges can serve as a passive method to reduce agglomeration within the nanocomposite, effectively increasing the available surface area for adsorption-an elementary step in the
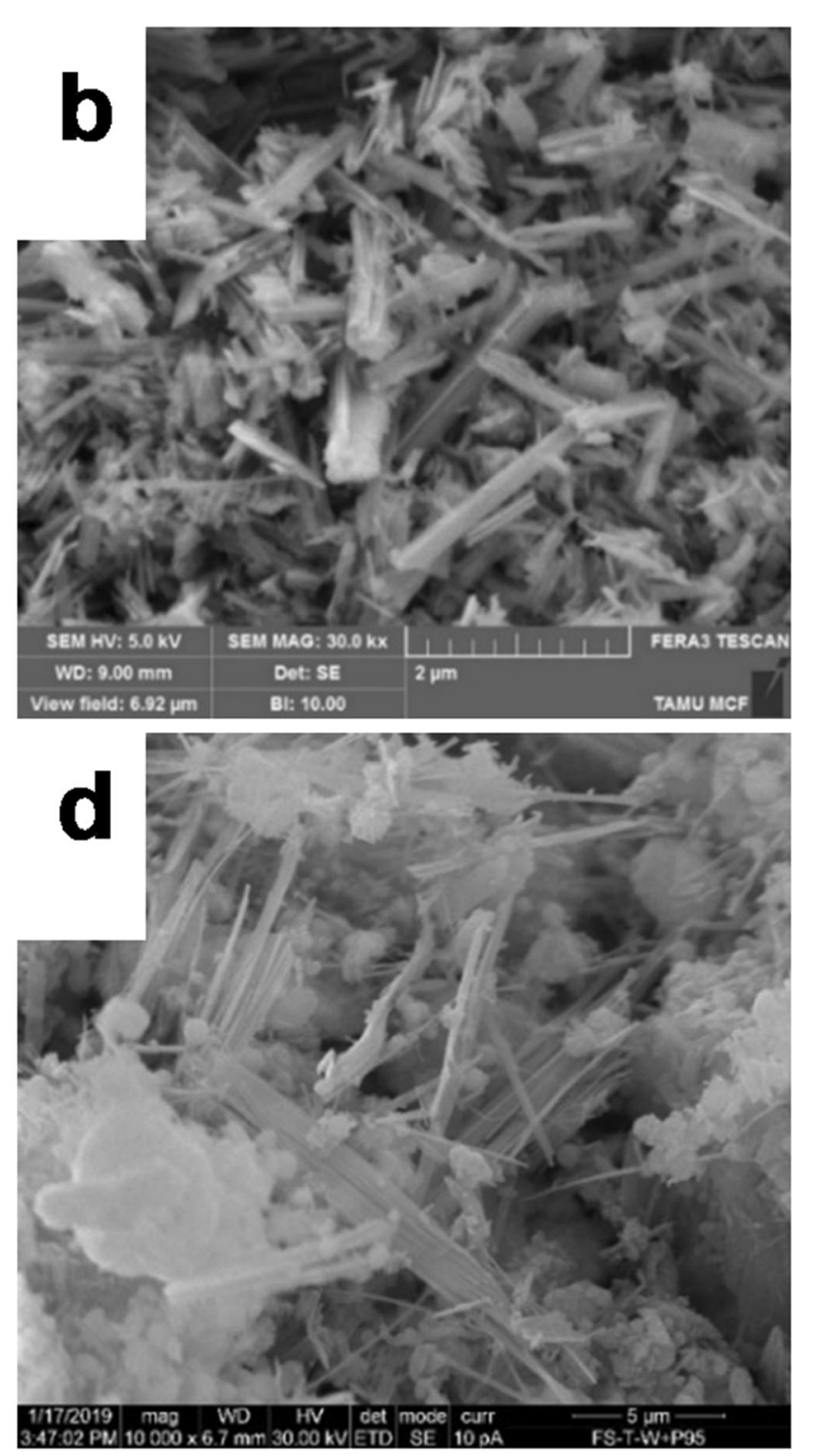


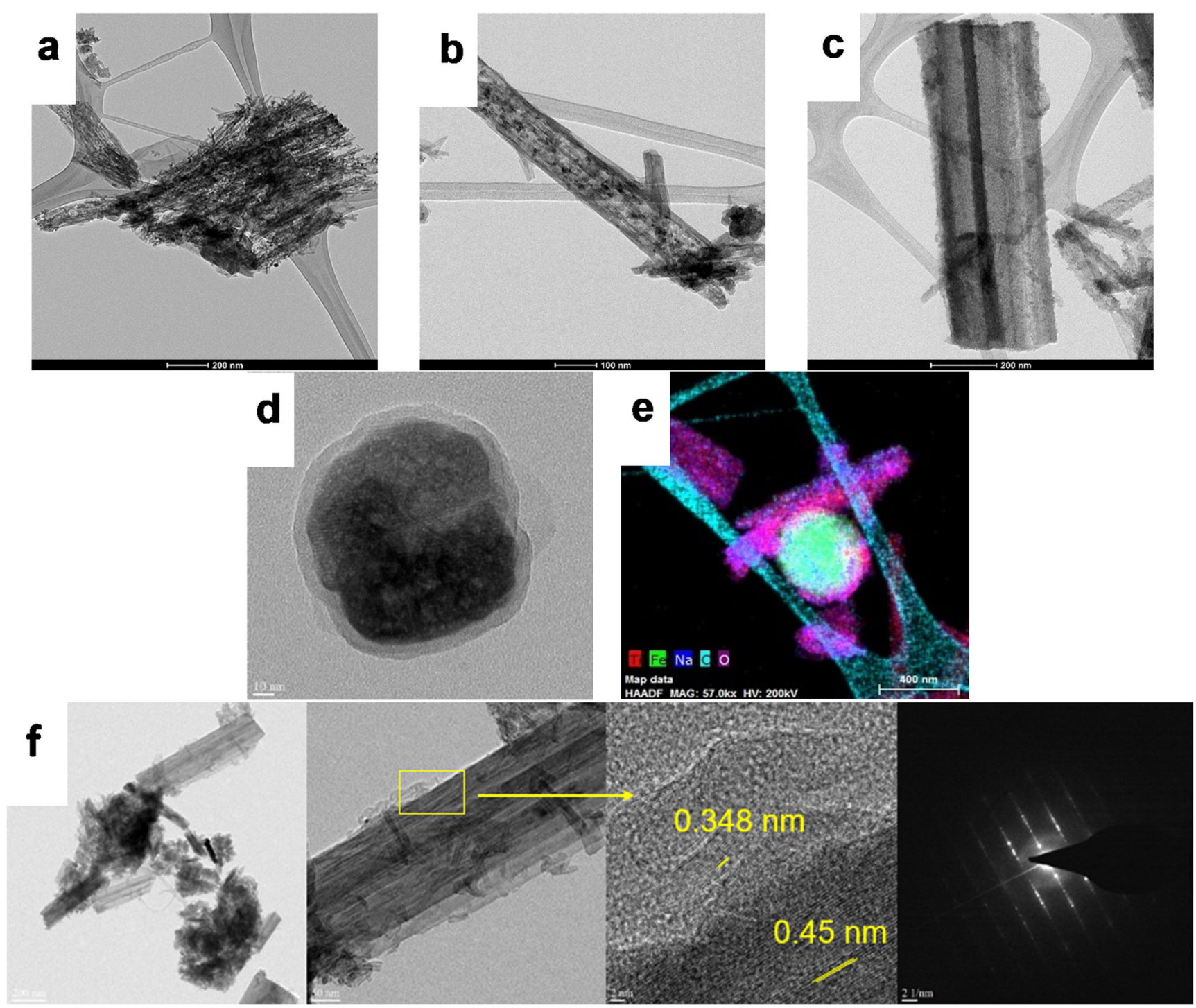

Fig. 3 Transmission electron microscopy (TEM) images: a pTNW/MWCNT (3); b pTNW/MWCNT (4); c bTNW/MWCNT; d SiO $@ \mathrm{MFe}_{3} \mathrm{O}_{4}$; e bTNW/MWCNT@ $\mathrm{SiO}_{2} @ \mathrm{Fe}_{3} \mathrm{O}_{4}$ (w/ additional P25); f Lattice fringe spacing of the bTNW/MWCNT photocatalyst

photochemical process. TEM imaging of pristine and reduced heterojunctioned titania-MWCNT samples in Fig. 3b and c shows an apparent maintenance of both morphology and heterojunctioning between titania and MWCNT. This ensures that the function of MWCNT as a charge carrier to indirectly reduce the relatively high electron-hole recombination rate of titania is in fact sustained upon reduction to black titania. A clear shadow atop the paramagnetic $\mathrm{Fe}_{3} \mathrm{O}_{4}$ microspheres shows the monodispersed silica layer in Fig. 3d. TEM elemental mapping of the final reduced paramagnetic nanocomposite in Fig. 3e shows the presence of the expected elements in a rather harmonious coupling of MWCNT with the main photocatalyst mesh. Elemental silicon was not detected, however, possibly due to analysis being performed on a location with low silica coverage atop the iron oxide core. Residual sodium is present due to the high adsorptive capacity of MWCNT of Na during hydrothermal treatment. Lastly, Fig.
$3 \mathrm{f}$ shows lattice fringe spacing of the $\mathrm{bTNW} / \mathrm{MWCNT}$ photocatalyst, with lattice spacing equaling $0.348 \mathrm{~nm}$ corresponding to (101) anatase $\mathrm{TiO}_{2}$.

\subsubsection{FTIR-ATR, diffuse reflectance spectroscopy, and X-ray photoelectron spectroscopy}

The obtained FTIR-ATR spectra of pristine and reduced TNW/MWCNT and the paramagnetic black TNW/MWCNT nanocomposite are presented in Fig. 4. The notable increase in the $\mathrm{O}-\mathrm{H}$ stretch at $3440 \mathrm{~cm}^{-1}$ between the pristine and reduced/black sample is indicative of the presence of oxygen vacancies and surface defects introduced during reduction. Although spectra in Fig. 4a and b show a clear presence and successful heterojunctioning between MWCNT functional groups and that of titania, an apparent decrease in aliphatic bending vibrations can be noted at its corresponding sharp 


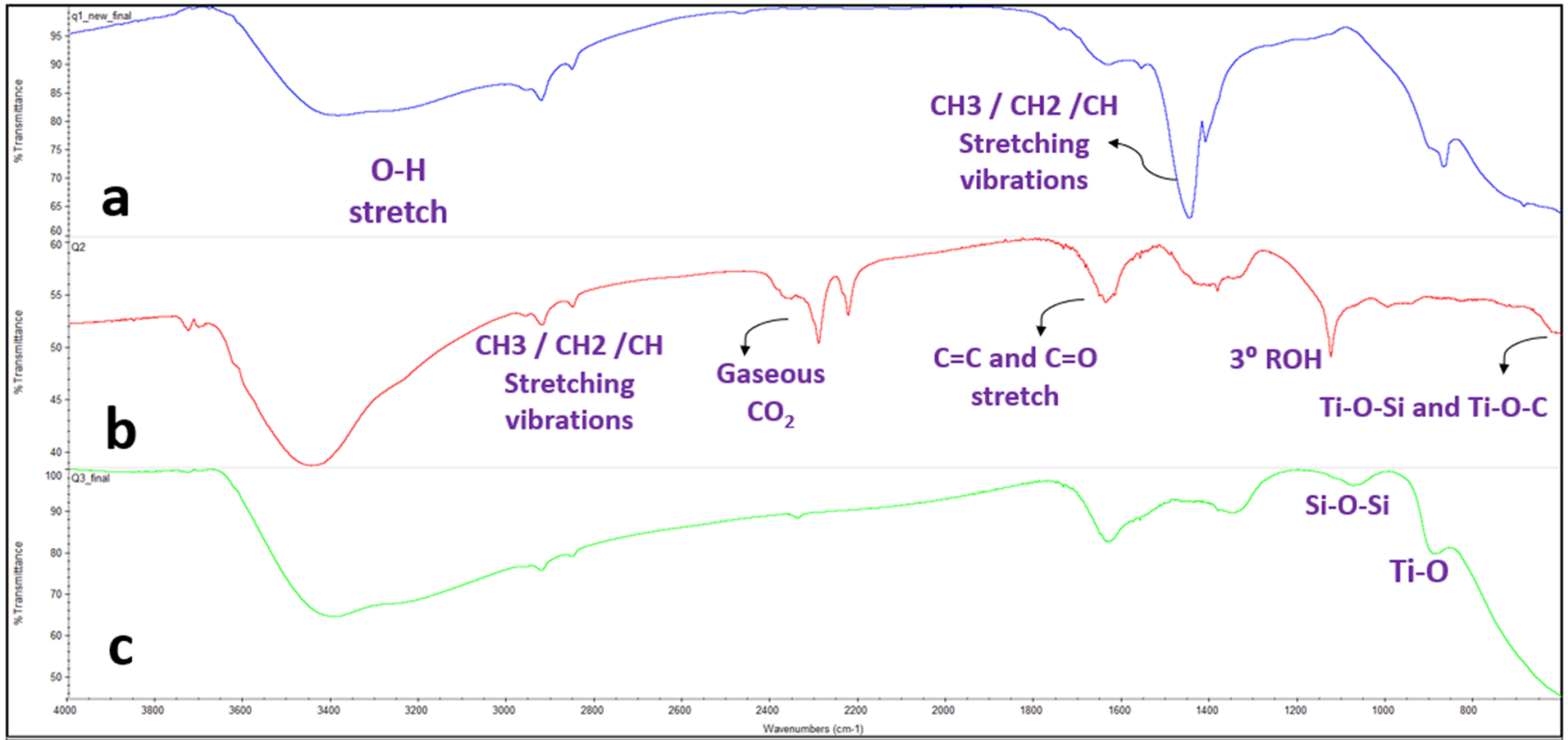

Fig. 4 FTIR-ATR spectra of a pTNW/MWCNT (4); b bTNW/MWCNT; c bTNW/MWCNT@ $\mathrm{SiO}_{2} @ \mathrm{Fe}_{3} \mathrm{O}_{4}$ (w/ additional P25)

peak at $1450 \mathrm{~cm}^{-1}$. The introduction of surface defects to the titania content present in the nanocomposite can inadvertently introduce some structural hindrances to the carbon allotrope, reducing bending vibration modes. This, however, is expected to have little to no effect on the apparent photoactivity of the reduced nanocomposite. Figure $4 \mathrm{~b}$ shows two characteristic peaks at $2250-2350 \mathrm{~cm}^{-1}$ range, which correspond to gaseous $\mathrm{CO}_{2}$ adsorbed through surface defects. Interestingly, Fig. $4 \mathrm{~b}$ also shows a substantial reduction in peak intensity in the $600-950 \mathrm{~cm}^{-1}$ range, corresponding to the $\mathrm{Ti}-\mathrm{O}$ and $\mathrm{Ti}-\mathrm{O}-$ $\mathrm{C}$ functional groups. Reduction in peak intensity in this area is highly indicative, and further confirms, the successful introduction of oxygen vacancies. No Ti-O and $\mathrm{Ti}-\mathrm{O}-\mathrm{C}$ bonds can form in the presence of an oxygen vacancy, since oxygen would be required in order to form these bonds. Figure $4 \mathrm{c}$ confirms the presence and effective heterojunctioning of the silica coating with the photoactive titania, as can be seen from the two peaks at $1095 \mathrm{~cm}^{-1}$ and $910 \mathrm{~cm}^{-1}$, which correspond to $\mathrm{Si}-\mathrm{O}-\mathrm{Si}$ bridge and antisymmetric $\mathrm{Ti}-\mathrm{O}-\mathrm{Si}$ bonds, respectively. The latter peak is broader than expected due to the overlapping of the heterojunctioning peak between titania and MWCNT as well.

Results of the UV-Vis diffuse reflectance spectra (DRS) show a clear trend in bandgap reduction as synthesis progresses from pristine titania spherical nanoparticles morphology, to reduced titania nanowire morphology with heterojunctioned MWCNT. Figure 5 shows pTNW ( $20 \mathrm{~h}$, no $\mathrm{EtOH}$, surface treatment (st), calcination) having a prominent redshift towards visible light compared with its P25 precursor. This is perhaps due to the introduction of some oxygen vacancies in the pTNW that were surface treated prior to calcination. Residual sulfate ions adsorbed to the titania surface prior to calcination can favorably form oxygen vacancies at these sites and in turn create trap energy states below the $\mathrm{CB}$

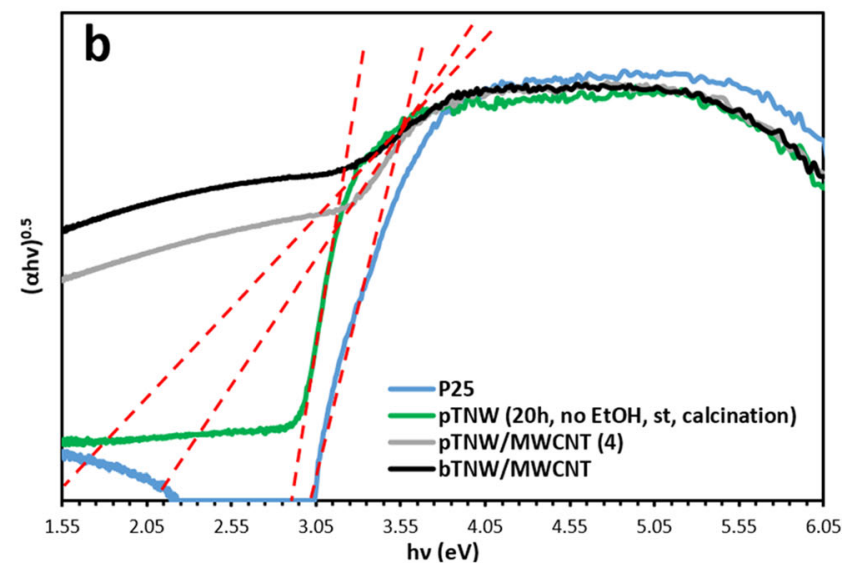

Fig. 5 a UV-Vis diffuse reflectance spectra (DRS); b Tauc's plot (Ahv) $)^{0.5}$ versus energy 
of anatase titania. Moreover, since anatase phase exhibits a slightly lower bandgap energy compared to rutile, it is safe to surmise that since the pTNW had a complete phase shift to anatase, a notable $0.15 \mathrm{eV}$ bandgap energy reduction from its constituent P25 precursor was also observed. Factoring the direct transition type semi-conductor nature of titania, optical bandgap energies were approximated using Tauc's equation [31]. It was found that the bandgap energies of the four pTNW/MWCNT solids had a discrepancy of about $0.7 \mathrm{eV}$, with pTNW/MWCNT (4) having the lowest bandgap energy of $2.08 \mathrm{eV}$. Since pTNW/MWCNT (4) was fabricated using pTNW as precursor and was surface-treated prior to calcination, it seems that a more effective heterojunctioning with the MWCNT allowed for a superior bandgap energy reduction. The reduced bTNW/MWCNT sample exhibited a superior response to visible light, and a bandgap energy of $1.45 \mathrm{eV}$

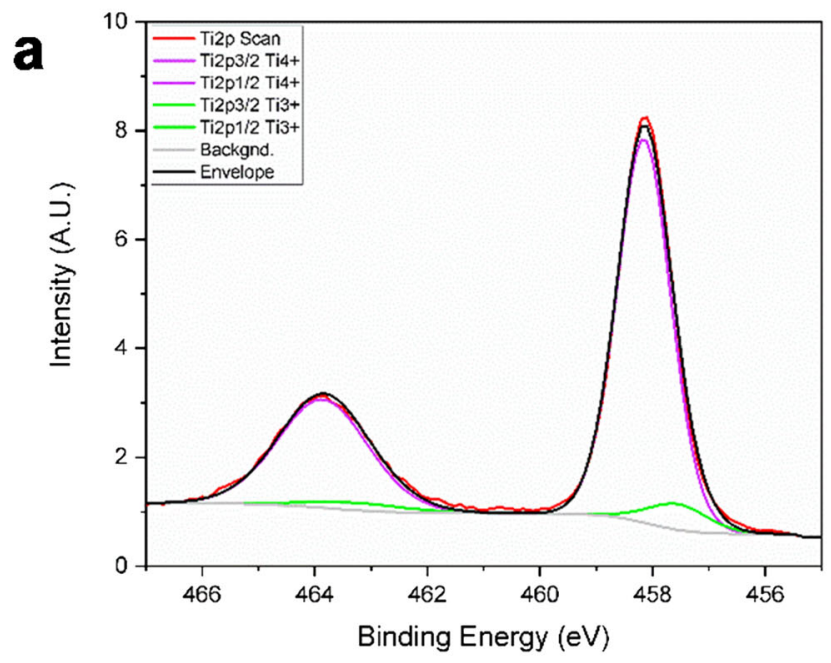

was approximated using Tauc's equation. The narrowing of the bandgap energy in bTNW/MWCNT can be attributed to the generation of donor energy states which reside below the $\mathrm{CB}$ - much like the narrowing of the bandgap upon carbon allotrope heterojunctioning and anatase phase properties.

XPS measurements were performed in order to probe the surface composition, and in particular the chemical states of elements in the as-prepared bTNW/MWCNT sample. Results of the high-resolution XPS spectra of Ti 2p, O 1s, and C 1s are shown in Fig. 6. The presence and further confirmation of oxygen vacancies are evident from the distinct deconvoluted peak of the Ti $2 p$ spectrum at $457.6 \mathrm{eV}$ and $463.4 \mathrm{eV}$, which correspond to $\mathrm{Ti}^{3+} 2 \mathrm{p}_{3 / 2}$ and $\mathrm{Ti}^{3+} 2 \mathrm{p}_{1 / 2}$, respectively, indicating the presence of $\mathrm{Ti}^{3+}$ states created via $\mathrm{NaBH}_{4}$ reduction. Discrete $\mathrm{Ti}^{4+}$ peaks are located at $458.2 \mathrm{eV}$ and $463.9 \mathrm{eV}$, corresponding to $\mathrm{Ti}^{4+} 2 \mathrm{p}_{3 / 2}$ and $\mathrm{Ti}^{4+} 2 \mathrm{p}_{1 / 2}$, respectively.

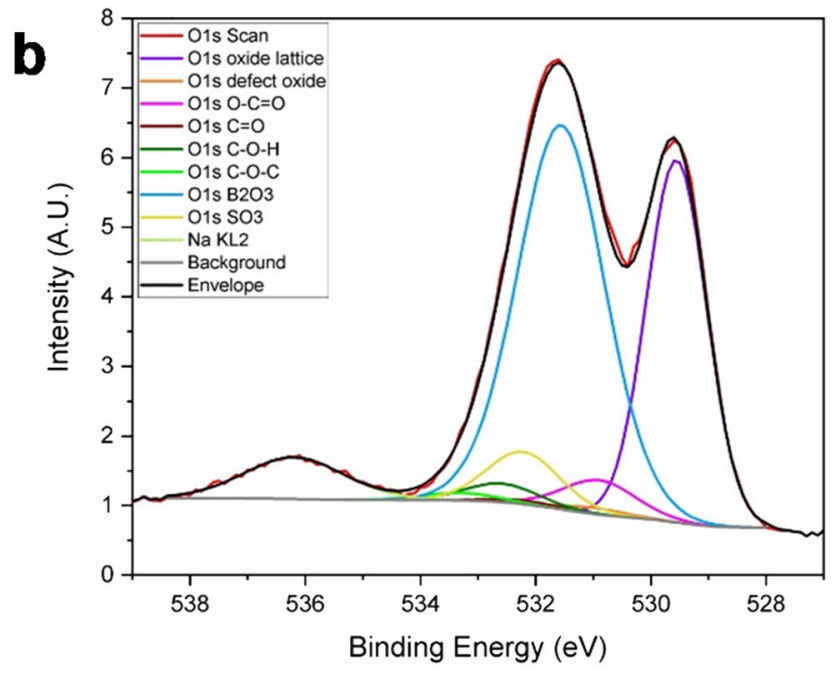

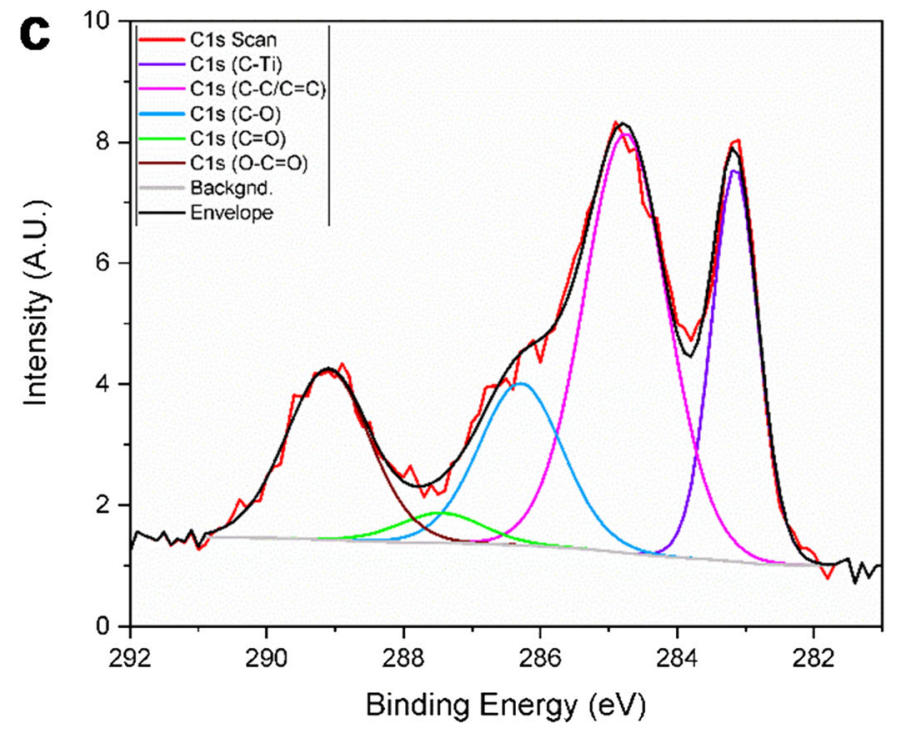

Fig. 6 XPS: a Ti2p spectra; b O1s spectra; c C1s spectra for the synthesized bTNW/MWCNT 
Furthermore, the $\mathrm{O}$ 1s spectra, shown in Fig. 6b, can be fitted into eight peaks, namely, the predominant $\mathrm{O}-\mathrm{H}$ group peak at $531.8 \mathrm{eV}$ and lattice $\mathrm{Ti}-\mathrm{O}$ peak at $529.5 \mathrm{eV}$, but also $\mathrm{O}-\mathrm{C}=\mathrm{O}$, $\mathrm{C}=\mathrm{O}, \mathrm{C}-\mathrm{OH}, \mathrm{C}-\mathrm{O}-\mathrm{C}, \mathrm{B}_{2} \mathrm{O}_{3}$, and $\mathrm{SO}_{3}$. Interestingly, the peak intensity of the $\mathrm{O}-\mathrm{H}$ group was found to be higher than that of lattice titania which is possibly due to the adsorption of moisture oxygen at surface defect sites. High-resolution XPS spectrum of the $\mathrm{C}$ 1s peaks is shown in Fig. 6c. Five distinctive peaks located at $283.3 \mathrm{eV}, 284.7 \mathrm{eV}, 286.4 \mathrm{eV}, 287.8 \mathrm{eV}$, and $289.1 \mathrm{eV}$ are assigned to $\mathrm{Ti}-\mathrm{C}, \mathrm{C}=\mathrm{C}, \mathrm{C}-\mathrm{O}, \mathrm{C}=\mathrm{O}$, and $\mathrm{O}-\mathrm{C}=\mathrm{O}$ bonds, respectively. The distinctive metal (i.e., titanium) carbide peak at $283.3 \mathrm{eV}$ is an indication of the strong heterojunction present between lattice titania and the electron acceptor carbon allotrope used. Ti-C related Ti percentage is much smaller than $\mathrm{TiO}_{2}$ because it is formed only at a small interface between the $\mathrm{TiO}_{2}$-NW and the MWCNT. The presence of $\mathrm{B}$ in two chemical states $\left(\mathrm{B}_{2} \mathrm{O}_{3}\right.$ and $\left.\mathrm{NaBH}_{4}\right)$ has been confirmed and most of $\mathrm{NaBH}_{4}$, which was employed in the sample preparation that has been oxidized to $\mathrm{B}_{2} \mathrm{O}_{3}$. Functional groups formation-especially those found in the $\mathrm{C} 1 \mathrm{~s}$ spectra-increases with temperature and by using acids such as sulfuric acid (i.e., elucidating on the $\mathrm{SO}_{3}$ peak).

\subsection{Evaluation of photocatalytic degradation of MO}

Photocatalytic degradation of methylene orange (MO) using the as-prepared photocatalysts was carried out under artificial sunlight at a measured intensity of $700 \mathrm{~W} / \mathrm{m}^{2}$, and comparative studies has been performed using commercial P25. The photolysis control experiment showed that no apparent $\mathrm{MO}$ degradation occurs without the presence of a dispersed photocatalyst. The obtained results are shown in Fig. 7, and these results indicate that adsorption plays a negligible role for synthesized samples without heterojunctioning to MWCNT. However, approximately $5 \%$ of the MO is adsorbed during the initial $30 \mathrm{~min}$ of the experiment before irradiation for MWCNT-incorporated samples.

The degradation efficiency of MO by P25, pTNW, bTNW, pTNW/MWCNT (4), and bTNW/MWCNT over the initial $20 \mathrm{~min}$ of artificial solar light were found to be $18.3 \%$, $15.7 \%, 47 \%, 90.7 \%$, and $97.4 \%$, respectively. The obtained results show a similar trend that samples with a higher anatase phase generally exhibited higher photoactivity. Moreover, a clear jump in photodegradation efficiency is observed between pristine and reduced TNW, and between stand-alone pTNW and its heterojunctioned counterpart-pTNW/ MWCNT (4). The photodegradation results reflect the importance of maintaining a predominantly exposed anatase phase, heterojunctioning between the photoactive titania and MWCNT, and the reduced titania that allows for further reduction in bandgap energy and offer more sites for adsorption due to surface defects. Interestingly, the paramagnetic nanocomposite counterparts for the pristine and reduced TNW/ MWCNT exhibited an average $7.5 \%$ reduction in photoactivity. The decrease in photoactivity is perhaps due to the innately higher aggregation that was observed, in effect exposing less of the photoactive anatase surface.

The apparent reaction rate constant, assuming pseudo-firstorder kinetics, has been evaluated by plotting the apparent pseudo-first-order linear transform $\operatorname{Ln}\left(\mathrm{C}_{0} / \mathrm{C}_{\mathrm{t}}\right)=\mathrm{f}(\mathrm{t})$ as shown in Fig. 8. The apparent reaction rate constant value $(k)$ for $\mathrm{P} 25$, pTNW, bTNW, pTNW/MWCNT (4), and bTNW/MWCNT are $0.0081 \mathrm{~min}^{-1}, 0.0068 \mathrm{~min}^{-1}, 0.0418 \mathrm{~min}^{-1}, 0.1087 \mathrm{~min}^{-1}$, and $0.1439 \mathrm{~min}^{-1}$, respectively. All linear fittings showed

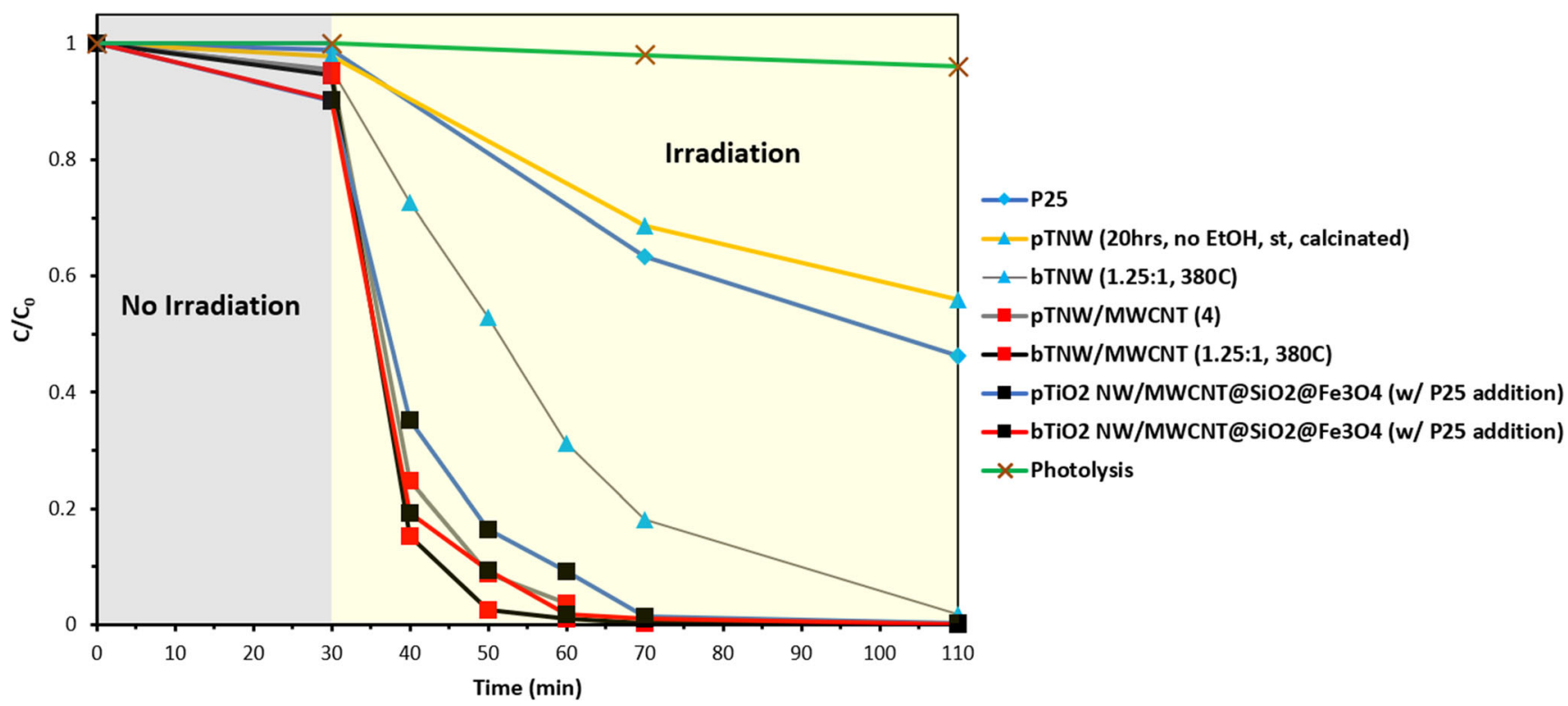

Fig. 7 Photocatalytic degradation of methylene orange (MO) aqueous solution over the synthesized photocatalysts (C is the corresponding degradative concentration of contaminant and $\mathrm{C}_{0}$ is the initial concentration of the contaminant) 
Fig. 8 Variations of $\operatorname{Ln}\left(\mathrm{C}_{0} / \mathrm{C}_{\mathrm{t}}\right)$ with respect to artificial solar light irradiation time $(\mathrm{C}$ is the corresponding degradative concentration of contaminant and $\mathrm{C}_{0}$ is the initial concentration of the contaminant)

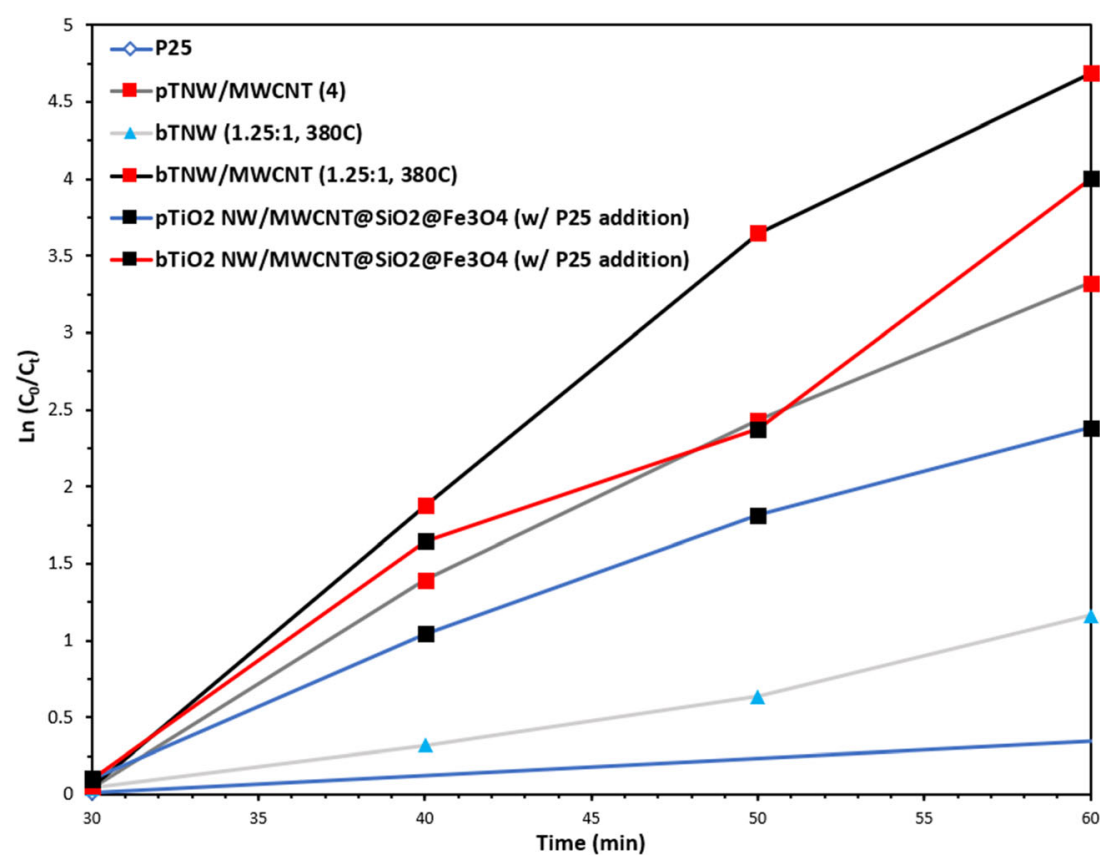

coefficient of determination $\left(R^{2}\right)$ values above 0.97 , showing that reaction kinetics can be represented by a pseudo-firstorder kinetic model. Furthermore, the $k$ value for bTNW/ MWCNT is significantly higher than commercial grade P25 (17.5 times higher), which is evident of the significant surface and structural modifications that were implemented during synthesis. In order to compare the effect of the two main modifications - namely, heterojunctioning with MWCNT and reduction - on photoactivity, the $k$ value between independent pTNW and its heterojunctioned equivalent shows 16 times increase in kinetics. Contrarily, the $k$ value between pristine and black TNW/MWCNT displays 1.3 times increase. Intriguingly, this shows that the successful heterojunctioning of titania with a charge carrier poses a more advantageous effect on photoactivity than does the introduction of surface defects by reduced titania. The separation of photogenerated carriers and the enhancement in electron transfer atop the chemically bound MWCNT allows for a significant reduction in the electron-hole recombination rate, accelerating photodegradation. The prepared catalyst compares well with recently reported photocatalysts for dye degradation. For instance, $\mathrm{Fe}_{3} \mathrm{O}_{4}-\mathrm{Ag}_{2} \mathrm{WO}_{4}$ degradation kinetics of fast green (FG) was recorded at $0.0107 \mathrm{~min}^{-1}, \mathrm{~N}-\mathrm{Pt}-\mathrm{TiO}_{2}$ PAMAM 0 exhibited $0.0044 \mathrm{~min}^{-1}$ kinetics in degrading brilliant black (BB), and PANI/SnS-0.5 reported $0.0404 \mathrm{~min}^{-1}$ first-order rate in visible light degradation of MO [32-34].

In order to probe an understanding on the optimum operating conditions of the developed photoactive material of the final paramagnetic nanocomposite, bTNW/MWCNT, the effects of $\mathrm{pH}$, catalyst loading, and initial concentration of MO were evaluated.
During these experiments, light intensity was maintained at $700 \mathrm{~W} / \mathrm{m}^{2}$, initial $\mathrm{MO}$ concentration was $10 \mathrm{mg} / \mathrm{L}$, catalyst load was $1 \mathrm{~g} / \mathrm{L}$, and initial $\mathrm{pH}$ was maintained at 7. All these experimental conditions were held constant for all experiments except the parameter that was evaluated as shown in Fig. 9. Also, the photocatalyst was evaluated for degradation of humic acid (HA) and methylene blue (MB), and the results were compared with the corresponding results for MO degradation. Figure 9a indicates that degradation rates at acidic and neutral $\mathrm{pH}$ values are almost similar and much faster than the rates at alkaline conditions. In an alkaline medium, surface crevices atop the photocatalyst's defect surface structure that have been introduced during reduction will contain hydroxyl ions. These ions repel the anionic MO molecules, in effect partially hindering adsorption and lowering successful photochemical oxidation events. Both catalyst dose and initial concentration of MO were found to have a negligible effect on the photodegradation performance, as shown in Fig. 9b and c. Moreover, Fig. 9d indicates that the developed photocatalyst material has effective and non-selective degradation progression with both anionic (MO) and cationic (MB) dyes alike. There is, however, a significant decrease in HA degradation rate. This can be attributed to the composition of the HA sodium salt used, which has less than 50\% HA purity. In this case, other intermediates present can impede photodegradation, or simply form intermediate complexes that are detected with the UV-Vis spectrophotometer under the same absorption band as residual HA concentrations. 

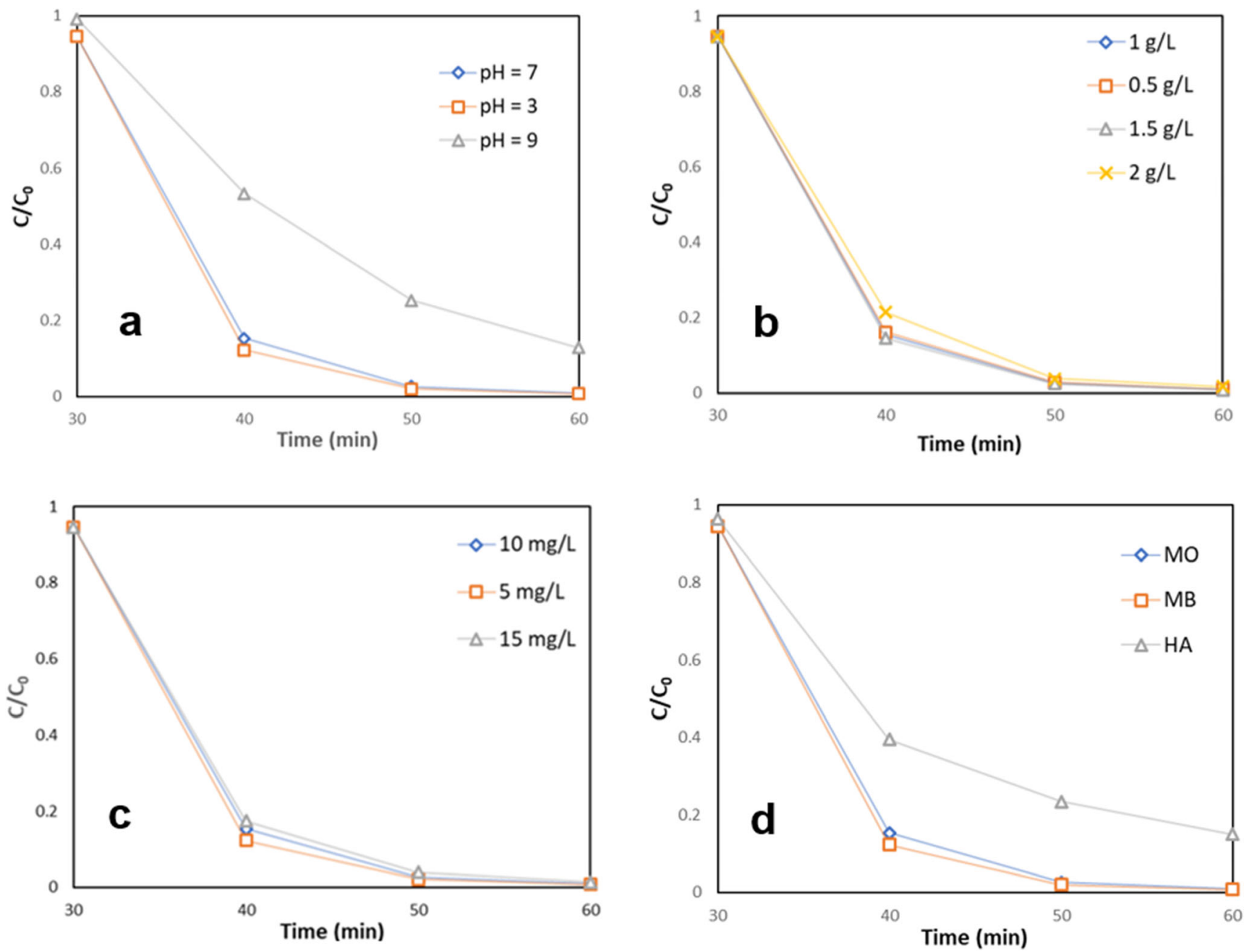

Fig. 9 Photocatalytic degradation of methylene orange (MO) aqueous solution over bTNW/MWCNT under a varying pH, b varying catalyst loads, $\mathbf{c}$ varying $\mathrm{MO}$ initial concentration, and $\mathbf{d}$ varying organic contaminants

\section{Conclusion}

In this work, successful design, fabrication, and characterization of high performance modified titania-based photocatalyst were achieved. The surface defects atop the titania nanostructure allowed for the utilization of both UV and visible light regions. Moreover, the establishment of a high ratio of oxygen vacancies on the titania structure, along with MWCNTs heterojunctions, led to a notable reduction in the high bandgap energy of pristine titania $(3.2 \mathrm{eV})$ and resulted in a bandgap energy of $1.45 \mathrm{eV}$ of the final photocatalyst. The promising photocatalyst developed, bTNWs/MWCNTs (1.25:1, 380C), yielded very high photodegradation efficiency $(97.4 \%)$ of MO in a mere $20 \mathrm{~min}$ of artificial solar irradiation of $700 \mathrm{~W} / \mathrm{m}^{2}$ light intensity.

A predominant anatase phase with (101) exposed lattice facets were confirmed from XRD and HRTEM lattice fringe spacings and were found to be of paramount importance. Approximations using Scherrer equation clearly showed that for crystallite size of $15 \mathrm{~nm}$, there is a decreasing dependence on crystallite size in terms of enhancing electron surface distance migration, and in effect decreasing the electron-hole recombination rate. Surface treatment procedures were found to have notable impacts on morphological roughening of surface and edge structures of 1dimensional titania nanostructures (i.e., nanowires), which in turn have had a phenomenal enhancement of photoactivity. The presence of oxygen vacancies and $\mathrm{Ti}^{3+}$ were found to further enhance photoactivity via the reduction in bandgap energy of titania. Surface defects introduced through reduction schemes were found to enhance adsorption and photoactivity of organic contaminants. Incorporating MWCNTs while ensuring for a successful heterojunction with the surface of the titania and low aggregation of the nanocomposite was found to be highly dependent on the morphology of titania precursor used. Wastewaters generated by textile and paper industries typically involve convoluted and multi-category molecules including organic dyes, intermediates, phenols and benzenes, and inorganic salts and heavy metals. Thus, future research on evaluating the attained photocatalyst for degrading emerging persistent contaminants in complex wastewater systems is warranted. 
Funding This research was supported by a grant from Texas A\&M University at Qatar under its Responsive Research Seed Grants (RRSG) Program. Open Access funding provided by the Qatar National Library.

\section{Compliance with ethical standards}

Conflict of interest The authors declare that they have no conflict of interest.

Open Access This article is licensed under a Creative Commons Attribution 4.0 International License, which permits use, sharing, adaptation, distribution and reproduction in any medium or format, as long as you give appropriate credit to the original author(s) and the source, provide a link to the Creative Commons licence, and indicate if changes were made. The images or other third party material in this article are included in the article's Creative Commons licence, unless indicated otherwise in a credit line to the material. If material is not included in the article's Creative Commons licence and your intended use is not permitted by statutory regulation or exceeds the permitted use, you will need to obtain permission directly from the copyright holder. To view a copy of this licence, visit http://creativecommons.org/licenses/by/4.0/.

\section{References}

1. M. Ge, C. Cao, J. Huang, S. Li, Z. Chen, K.Q. Zhang, et al., A review of one-dimensional $\mathrm{TiO} 2$ nanostructured materials for environmental and energy applications. J. Mater. Chem. A 4(18), 6772-6801 (2016)

2. Lacombe, S., Fresno, F., \& Štangar, U. L., Photocatalysis: new highlights from JEP 2013. 2014

3. F.M. Hossain, A.V. Evteev, I.V. Belova, J. Nowotny, G.E. Murch, Electronic and optical properties of anatase $\mathrm{TiO} 2$ nanotubes. Comput. Mater. Sci. 48(4), 854-858 (2010)

4. R. Chalasani, S. Vasudevan, Cyclodextrin-functionalized Fe3O4@ $\mathrm{TiO} 2$ : reusable, magnetic nanoparticles for photocatalytic degradation of endocrine-disrupting chemicals in water supplies. ACS Nano 7(5), 4093-4104 (2013)

5. H. Liu, Z. Jia, S. Ji, Y. Zheng, M. Li, H. Yang, Synthesis of TiO2/SiO2@Fe3O4 magnetic microspheres and their properties of photocatalytic degradation dyestuff. Catal. Today 175(1), 293 298 (2011)

6. T. Xin, M. Ma, H. Zhang, J. Gu, S. Wang, M. Liu, Q. Zhang, A facile approach for the synthesis of magnetic separable Fe3O4@ $\mathrm{TiO} 2$, core-shell nanocomposites as highly recyclable photocatalysts. Appl. Surf. Sci. 288, 51-59 (2014)

7. R. Asahi, T. Morikawa, T. Ohwaki, K. Aoki, Y. Taga, Visible-light photocatalysis in nitrogen-doped titanium oxides. Science 293(5528), 269-271 (2001)

8. D.R. Burri, K.M. Choi, S.C. Han, A. Burri, S.E. Park, Selective conversion of ethylbenzene into styrene over $\mathrm{K} 2 \mathrm{O} / \mathrm{TiO} 2-\mathrm{ZrO} 2$ catalysts: Unified effects of $\mathrm{K} 2 \mathrm{O}$ and $\mathrm{CO} 2$. J. Mol. Catal. A Chem. 269(1-2), 58-63 (2007)

9. G.S. Pozan, A. Kambur, Significant enhancement of photocatalytic activity over bifunctional $\mathrm{ZnO}-\mathrm{TiO} 2$ catalysts for 4-chlorophenol degradation. Chemosphere 105, 152-159 (2014)

10. B.K. Vijayan, N.M. Dimitrijevic, J. Wu, K.A. Gray, The effects of Pt doping on the structure and visible light photoactivity of titania nanotubes. J. Phys. Chem. C 114(49), 21262-21269 (2010)

11. X. Chen, L. Liu, Y.Y. Peter, S.S. Mao, Increasing solar absorption for photocatalysis with black hydrogenated titanium dioxide nanocrystals. Science 331(6018), 746-750 (2011)
12. C. Zhang, H. Yu, Y. Li, Y. Gao, Y. Zhao, W. Song, et al., Supported noble metals on hydrogen-treated $\mathrm{TiO} 2$ nanotube arrays as highly ordered electrodes for fuel cells. ChemSusChem 6(4), 659-666 (2013)

13. X. Liu, S. Gao, H. Xu, Z. Lou, W. Wang, B. Huang, Y. Dai, Green synthetic approach for $\mathrm{Ti} 3+$ self-doped $\mathrm{TiO} 2-\mathrm{x}$ nanoparticles with efficient visible light photocatalytic activity. Nanoscale 5(5), 1870-1875 (2013)

14. C. Xu, Y. Song, L. Lu, C. Cheng, D. Liu, X. Fang, et al., Electrochemically hydrogenated $\mathrm{TiO} 2$ nanotubes with improved photoelectrochemical water splitting performance. Nanoscale Res. Lett. 8(1), 1-7 (2013)

15. A. Badreldin, A.E. Abusrafa, A. Abdel-Wahab, Oxygen deficient cobalt-based oxides for electrocatalytic water splitting. ChemSusChem 14(1), 10-32 (2021)

16. A. Badreldin, A.E. Abusrafa, A. Abdel-Wahab, Oxygen-deficient perovskites for oxygen evolution reaction in alkaline media: a review. Emergent Mater. 3, 567-590 (2020)

17. Z. Li, B. Gao, G.Z. Chen, R. Mokaya, S. Sotiropoulos, G.L. Puma, Carbon nanotube/titanium dioxide (CNT/TiO2) core-shell nanocomposites with tailored shell thickness, CNT content and photocatalytic/photoelectrocatalytic properties. Appl. Catal. B Environ. 110, 50-57 (2011)

18. C.Y. Kuo, Prevenient dye-degradation mechanisms using UV/TiO2/ carbon nanotubes process. J. Hazard. Mater. 163(1), 239-244 (2009)

19. C. Hu, R. Zhang, J. Xiang, T. Liu, W. Li, M. Li, et al., Synthesis of carbon nanotube/anatase titania composites by a combination of sol-gel and self-assembly at low temperature. J. Solid State Chem. 184(5), 1286-1292 (2011)

20. S. Aryal, C.K. Kim, K.W. Kim, M.S. Khil, H.Y. Kim, Multi-walled carbon nanotubes/TiO2 composite nanofiber by electrospinning. Mater. Sci. Eng. C 28(1), 75-79 (2008)

21. S. Muduli, W. Lee, V. Dhas, S. Mujawar, M. Dubey, K. Vijayamohanan, et al., Enhanced conversion efficiency in dyesensitized solar cells based on hydrothermally synthesized $\mathrm{TiO} 2-$ MWCNT nanocomposites. ACS Appl. Mater. Interfaces 1(9), 2030-2035 (2009)

22. H. Yu, X. Quan, S. Chen, H. Zhao, Y. Zhang, TiO2-carbon nanotube heterojunction arrays with a controllable thickness of $\mathrm{TiO} 2$ layer and their first application in photocatalysis. J. Photochem. Photobiol. A Chem. 200(2-3), 301-306 (2008)

23. G. Rao, K.S. Brastad, Q. Zhang, R. Robinson, Z. He, Y. Li, Enhanced disinfection of Escherichia coli and bacteriophage MS2 in water using a copper and silver loaded titanium dioxide nanowire membrane. Front. Environ. Sci. Eng. 10(4) (2016)

24. J. Tian, X. H, H. Yang, Y. Zhou, H. Cui, H. Lui, High yield production of reduced $\mathrm{TiO} 2$ with enhanced photocatalytic activity. Appl. Surf. Sci. 360, 738-743 (2016)

25. A. Badreldin, A. Abdel-Wahab, P.B. Balbuena, Local surface modulation activates metal oxide electrocatalyst for hydrogen evolution: synthesis, characterization, and DFT study of novel black $\mathrm{ZnO}$. ACS Appl. Energy Mater. 3(11), 10590-10599 (2020)

26. D. Ariyanti, L. Mills, J. Dong, Y. Yao, W. Gao, NaBH4 modified TiO2: Defect site enhancement related to its photocatalytic activity. Mater. Chem. Phys. 199, 571-576 (2017)

27. X. Yu, S. Liu, J. Yu, Superparamagnetic $\gamma-\mathrm{Fe} 2 \mathrm{O} 3 @ \mathrm{SiO} 2 @ \mathrm{TiO} 2$ composite microspheres with superior photocatalytic properties. Appl. Catal. B Environ. 104(1-2), 12-20 (2011)

28. X. Song, L. Gao, Fabrication of hollow hybrid microspheres coated with silica/titania via sol- gel process and enhanced photocatalytic activities. J. Phys. Chem. C 111(23), 8180-8187 (2007)

29. R. Trofimovaite, C.M. Parlett, S. Kumar, L. Frattini, M.A. Isaacs, K. Wilson, et al., Single atom $\mathrm{Cu}$ (I) promoted mesoporous titanias for photocatalytic methyl orange depollution and $\mathrm{H} 2$ production. Appl. Catal. B Environ. 232, 501-511 (2018) 
30. B. Ingham, M.F. Toney, in Metallic Films for Electronic, Optical and Magnetic Applications, ed. By K. Barmak, K. Coffey (Woodhead Publishing, 2014), pp. 3-38.

31. A.S. Hassaniena, A.A. Akl, Effect of Se addition on optical and electrical properties of chalcogenide CdSSe thin films. Superlattice. Microst. 89, 153-169 (2016)

32. S. Rajamohan, V. Kumaravel, R. Muthuramalingam, S. Ayyadurai, A. Abdel-Wahab, B.S. Kwak, et al., Fe 3 O 4-Ag 2 WO 4: facile synthesis, characterization and visible light assisted photocatalytic activity. New J. Chem. 41(20), 11722-11730 (2017)
33. S.K. Nzaba, H.H. Nyoni, B.B. Mamba, A.T. Kuvarega, Comparative study of dendrimer-templated nitrogen-platinum codoped $\mathrm{TiO} 2$ for the photocatalytic degradation of Azo dyes in contaminated water. ChemistrySelect 4(41), 12156-12163 (2019)

34. K. Yao, Y. Liu, H. Yang, J. Yuan, S. Shan, Polyaniline-modified 3D-spongy SnS composites for the enhanced visible-light photocatalytic degradation of methyl orange. Colloids Surf. A Physicochem. Eng. Asp. 603, 125240 (2020) 